\title{
Assessing Transformation: Findings from the Measuring Reuse Project
}

\author{
Santi Thompson \\ University of Houston, USA \\ Liz Woolcott \\ Utah State University, USA \\ Caroline Muglia \\ University of Southern California, USA \\ Genya O'Gara \\ Virtual Library of Virginia, USA
}

Ayla Stein Kenfield

University of Illinois at Urbana-Champaign, USA

Elizabeth Joan Kelly

Loyola University New Orleans, USA

\section{Introduction}

Cultural heritage and knowledge organizations (CHKOs) have been digitizing analog content and acquiring born-digital materials for decades. Large, complex digital repositories and aggregated websites now make countless historic images, manuscripts, audio/video, research datasets, and other kinds of resources more widely available than ever before. Over time institutions have found multiple benefits for expanding access to rare and unique materials, including enhancing scholarship, promoting preservation, and increasing usage.

While the profession continues to develop extensive workflows and tools to facilitate online access to content, the assessment of these practices and the digital collections they yield has been inconsistent and incomplete. Researchers interested in refining digital repository software have spent significant time on user experience studies; others have also tried to understand, standardize, and quantify general usage practices among patron audiences. Another specific evaluation criteria-the ability to measure and assess how digital objects are reused by various user groups-has also emerged in the professional conversation and literature. Refining how practitioners evaluate reuse, or how a digital object is utilized or repurposed, offers an opportunity to identify and standardize techniques that can improve the ways that CHKOs measure and define impact. ${ }^{1}$

The authors of this paper viewed these assessment gaps as barriers for CHKOs that needed to be addressed. They believe that the development of a reuse assessment toolkit would be a valuable resource for digital library practitioners. Relying on feedback from a wide range of digital library practitioners, the authors spent one year compiling data from survey results and focus group interviews to identify the needs and ideal functionality of a future digital object reuse assessment toolkit. This project, titled "Developing a Framework for Measuring Reuse of Digital Objects," (hereafter "Measuring Reuse") was made possible by an Institute of Museum and Library Services (IMLS) National Leadership/National Forum grant.

This paper addresses the findings of the needs assessment. Specifically, it outlines the results and implications of a community follow-up survey that rated and prioritized use cases and functional 
requirements for the future assessment toolkit. Drawing upon data generated by the grant project, the authors ask two questions:

1. What are the prioritized use cases for a future reuse assessment toolkit?

2. What are the prioritized functional requirements for a future reuse assessment toolkit that would meet practitioner needs?

The results of this paper compliment a previous publication that outlined the results of other grant-related data collecting efforts, "Barriers and solutions to assessing digital library reuse: preliminary findings" (hereafter "Barriers and solutions"). ${ }^{2}$ Combined, these publications provide one of the profession's first indepth investigations of digital repository reuse assessment frameworks.

\section{Background}

Members of the Measuring Reuse project team are also members of the Content Reuse Working Group (CRWG), ${ }^{3}$ which is part of a larger, organized assessment effort: the Digital Library Federation (DLF) Assessment Interest Group (AIG). ${ }^{4}$ Founded in 2014, the DLF AIG aims to collaborate with CHKO communities to develop best practices and guidelines for various kinds of digital repository assessment. As part of the larger AIG, the CRWG has been focused on understanding the current state of digital library reuse assessment and developing tools and strategies to assist practitioners in their assessment efforts.

In 2015, members of the CRWG released "Surveying the Landscape: Use and Usability Assessment of Digital Libraries" (hereafter "Surveying the Landscape"). ${ }^{5}$ This white paper outlined the challenges institutions face in assessing digital repository content reuse. It discussed how library analytics focus almost entirely on simple access metrics. These types of statistics do not provide a nuanced picture of how users consume or transform unique materials from digital collections hosted by CHKOs. This lack of distinction, combined with a lack of standardized assessment approaches, makes it difficult for institutions to develop userresponsive collections or highlight the value of these materials. This in turn presents significant challenges for developing the appropriate staffing, system infrastructure, and long-term funding models needed to support digital collections. ${ }^{6}$

\section{Definitions}

As part of the project, the authors developed working definitions for both use and reuse. They generated this information to help participants understand the parameters of reuse as well as to distinguish the differences between digital object use and reuse. They situated use as the process of accessing particular content. Measuring use does not necessarily require a practitioner to have a nuanced understanding of how a user engages with any particular object. Often knowing that a user has "visited" or "downloaded" an object satisfies evaluation criteria for this category. On the other hand, reuse draws upon a nuanced understanding of how an object is repurposed or transformed. While these concepts, definitions, and examples are in a state of flux, the authors draw upon these terms for the purposes of this paper. The authors intend to expand upon and refine these definitions during future research. 


\begin{tabular}{|l|l|l|}
\hline Function & Definition & Examples \\
\hline Use & $\begin{array}{l}\text { Discovering and browsing } \\
\text { objects in a digital library, often } \\
\text { described as “clicks" or } \\
\text { "downloads," without knowing } \\
\text { the specific context for this use. }\end{array}$ & $\begin{array}{l}\text { Basic web usage analytics such as number of } \\
\text { downloads (unmediated), web traffic } \\
\text { information, IP address location, referrals } \\
\text { from specific sites, etc. }\end{array}$ \\
\hline Reuse & $\begin{array}{l}\text { How often and in what ways } \\
\text { digital library materials are } \\
\text { utilized and repurposed. In this } \\
\text { definition, a practitioner does } \\
\text { know the context of the use. }\end{array}$ & $\begin{array}{l}\text { information, citation metrics of data and/or } \\
\text { digital collection materials, inclusion of digital } \\
\text { collection materials in an external dataset } \\
\text { (e.g., HTRC datasets or curated Internet } \\
\text { Archive user collections), remixing songs, } \\
\text { mashups of two or more songs, creating } \\
\text { memes, sharing content on social media. }\end{array}$ \\
\hline
\end{tabular}

\section{Literature Review}

The authors' first article on this grant project addressed many of the current case studies and developments related to digital library use and reuse assessment. ${ }^{7}$ Consequently, this literature review will only briefly review some of the contributions highlighted in "Barriers and solutions" as well as focus on three recently published articles that address broad aspects of digital repository and content reuse assessment frameworks.

"Barriers and solutions" drew upon the major findings from the 2015 white paper, "Surveying the Landscape," to provide an overview of content reuse assessment between 2011 and 2015. "Surveying the Landscape" suggested that increased attention was being paid to reuse assessment for targeted audiences and disciplines, specifically humanities and the arts. ${ }^{8}$ However, the white paper also pointed out deficiencies-including difficulties tracking reuse through hyperlinking and measuring reuse from virtual to analog environments. ${ }^{9}$ Subsequent efforts further refined the profession's understanding of reuse assessment. "Barriers and solutions" reviews the movement within the digital library community to establish and implement "standards that demonstrate the impact of repositories," including Jisc's Institutional Repository Usage Statistics (IRUS) UK program and Montana State University's Repository Analytics \& Metrics Portal (RAMP)..$^{10}$ It also mentions recent practices used by information professionals to track and measure reuse over the web, including the use of reverse image lookup technology, Wikipedia citations, Google alerts, and embedded metadata. ${ }^{11}$

Beyond these advancements, several recent articles have tackled the challenge of formulating analytical frameworks for assessing reuse. "Barriers and solutions" reviewed qualitative measures articulated in "Beyond Clicks, Likes, and Downloads: Identifying Meaningful Impacts for Digitized Ethnographic Archives." Researchers in this study devised a framework for "documenting, demonstrating, and assessing the impact of digitized ethnographic collections." ${ }^{2}$ They formulated six topical areas of potential impact, including: knowledge, professional discourse, attitudes, institutional capacity, policy, and relationships. They note that these areas can assist how "institutions and communities articulate and assess major sorts of impact that are most relevant to institutional projects to digitize and share knowledge."13

In "Multifaceted Evaluation Criteria of Digital Libraries in Academic Settings: Similarities and Differences from Different Stakeholders," Iris Xie, Soohyung Joo, and Krystyna K. Matusiak seek to understand which digital library assessment criteria and frameworks are important to three different stakeholders: digital library (DL) scholars, practitioners, and users. While the researchers hypothesized that there would be "no 
significant difference in rating each evaluation criterion of each DL evaluation dimension among the three group of stakeholders," ${ }^{4}$ they found that significant differences did exist. The practitioner group ranked preservation assessment criteria as the most important, while DL scholars and users ranked nearly all categories as important. ${ }^{15}$ Xie et al.'s work suggests that assessment criteria and frameworks still remain under considerable flux among the stakeholders who study, interact with, and manage repositories.

Finally, the authors' own article, "Barriers and solutions," details one of the most comprehensive analyses of content reuse assessment frameworks. The article recounts the multiple approaches included in the authors' needs assessment and emphasized results from two data collection activities: an initial survey and focus groups. ${ }^{16}$ Analyzing the results of these activities suggested to the authors that an emergent need "is looking for field-wide approaches for assessing the impact of reuse in order to better understand, and tell the story of, what has been learned or gained by a user when they repurpose a digital object." ${ }^{17}$ However, "Barriers and solutions" was published before the authors could analyze another key data collection method-the results of a follow-up survey-which asked DL practitioners to prioritize emerging use cases and functional requirements. "Assessing Transformation" incorporates the prioritized use cases and functional requirements, providing a fuller picture of the authors' results, as well as providing the profession with a more nuanced view of emerging needs for a content reuse assessment framework.

\section{Methodology}

As outlined in the project grant proposal, the purpose of this research study was to "conduct a robust digital cultural heritage community needs assessment focused on developing use cases and functional requirements for a future digital content reuse assessment toolkit." ${ }^{18}$ The project team's approach for assessing the community's needs for a toolkit was constructed around two surveys (a pre- and post-survey) and a series of focus groups. The initial survey, administered September through October 2017, queried the community about their use and reuse assessment practices as well as investigated barriers and complications for conducting assessment of digital repository content. The results of this survey fed into the structure and questions for ten focus groups, which were designed around three primary topics: exploratory information gathering, technology and standards, and cultural issues and privacy. Focus groups were conducted from December 2017 through June 2018. Also during June 2018, the final follow-up survey was conducted. This survey pulled together use cases identified from the focus groups and asked the CHKO community to prioritize both the use cases and also the functional requirements of a reuse assessment toolkit.

\section{Administration and Analysis}

Both surveys were built and distributed using the Qualtrics survey platform. They were shared on 25 email lists and multiple social media platforms for digital repository practitioners. Responses were analyzed primarily using the Qualtrics platform analytics, but also included the analytics available in Google Sheets and Microsoft Excel. The survey analyses were compiled into reports that reflected the aggregated responses to each question as well as cross-tabulations to show patterns. Each report included a detailed summary and a set of recommendations. The survey instruments, datasets, and analytical reports for both surveys can be found on the project team's OSF repository. ${ }^{19}$

As previously mentioned, the focus groups were organized around the three topics of information gathering, technology and standards, and cultural and privacy issues. Focus groups were conducted both in-person and virtually for each topic and averaged about four people per session. For the information gathering and the technology and standards focus groups, two sessions of in-person meetings and two sessions of virtual meetings were held. Due to time and budget constraints, only one in-person session and one virtual session were held to explore cultural and privacy issues, although these concerns were also discussed in the first two topical sessions. In total, 40 participants took part in the focus groups. 


\begin{tabular}{|l|l|l|l|l|l|}
\hline Focus Group & Type & Sessions & When & Participants & Topic \\
\hline Group 1 & In-person & 2 & October 2017 & 9 & $\begin{array}{l}\text { Information } \\
\text { Gathering }\end{array}$ \\
\hline Group 1 & Virtual & 2 & December 2017 & 7 & $\begin{array}{l}\text { Information } \\
\text { Gathering }\end{array}$ \\
\hline Group 2 & In-person & 2 & February 2018 & 8 & $\begin{array}{l}\text { Technology \& } \\
\text { Standards }\end{array}$ \\
\hline Group 2 & Virtual & 2 & March 2018 & 7 & $\begin{array}{l}\text { Technology \& } \\
\text { Standards }\end{array}$ \\
\hline Group 3 & In-person & 1 & April 2018 & 5 & Cultural \& Privacy \\
\hline Group 3 & Virtual & 1 & June 2018 & 4 & Cultural \& Privacy \\
\hline
\end{tabular}

In-person focus group sessions were held in three locations where prominent conferences were taking place, including the Digital Library Federation Forum which took place in Pittsburgh, Pennsylvania, in October $2017,{ }^{20}$ the Code4Lib conference which took place in Washington, DC, in February $2018,{ }^{21}$ and the Personal Digital Archiving Conference which took place in Houston, Texas, in April 2018. ${ }^{22}$ The virtual sessions were arranged through Zoom, an online conferencing system maintained by the Digital Library Federation.

All focus group sessions were conducted by two project team members, with one serving as facilitator and the other as the note-taker. Prior to each focus group, participants were sent the discussion questions to consider. All sessions were recorded in order to review and verify the content of the notes that were taken, with the audio recording destroyed after 48 hours to preserve the anonymity of the participants. No personally identifiable information was retained in focus group notes.

Results from all 10 sessions were analyzed using Dedoose, ${ }^{23}$ a qualitative data analysis tool, and an open coding process which was broken into three distinct phases: initial coding built from tagging central discussion topics, focused coding where tags and related excerpts from notes were categorized into broader themes, and finally the development of use cases from those themes. Use cases were grouped into three topics: (1) Data Collection, Analysis and Reporting, (2) Collection Development, and (3) Privacy, Rights Management, and Ethics, and were presented to the CHKO community in a follow-up survey.

Respondents to the survey were asked to rank and prioritize the use cases in each functional group. They were also asked to identify the most desirable functional requirements for a toolkit. The responses from the survey were analyzed to cull out the most important use cases to the CHKO community as well as the most useful toolkit functional requirements. Additional cross-tabulations were done to isolate which functional requirements supported the most highly ranked use cases in general, as well as which use cases and functional requirements best met the needs of institutions supporting underserved populations. 


\section{Results}

The results of the first survey were analyzed in detail and published in the article "Barriers and solutions," 24 as well as in "Measuring Reuse of Digital Objects: Preliminary Findings from the IMLS-funded project" as part of the 2018 conference proceedings for the Joint Conference on Digital Libraries (JCDL). ${ }^{25}$ Therefore, this paper will only summarize the results of those findings ${ }^{26}$ and concentrate the bulk of this analysis on the findings from the focus groups and follow-up survey.

The foundational survey found that most respondents (80\%) were engaging in some form of use assessment and tended to use tools such as Google Analytics or the statistics provided by their content management system platform. They gathered information on the number of visitors, downloads, and clicks. Only a minority of respondents $(40 \%)$ were actively gathering reuse data and they reported using tools and techniques such as social media metrics, alert services, and reverse image lookup. The two most commonly gathered data points were the number of citations and objects cited in scholarship as well as the number of published or reposted digital objects in digital media (such as online websites, digital exhibits, films or online videos, and subject-specific repositories). Respondents reported that they were generally very interested in assessment but would need support such as documented standards and recommended practices, more personnel, online tutorials, open source tools, and money. They also noted that they were interested in understanding more about the demographics of their users and how to turn assessment data into impact statements. $^{27}$

\section{Focus Groups}

Following some of the comments and free text answers presented in the survey, the themes for the focus groups were established to collect more detailed information. Each focus group built on the body of response data that came before it in order to develop an iterative and dynamic understanding of practitioner needs.

\section{Information-Gathering Focus Groups}

During the first set of focus groups, participants explored current practices for assessment of use or access and how it differed from reuse assessment; which stakeholders were important to consider for assessment data; barriers to both use and reuse assessment; types of use and reuse assessment data; technological issues with assessment; organizational and administrative support of assessment; use cases for showing impact, and; potential ways that reuse assessment could be controversial.

The themes that emerged from this first, exploratory focus group included the heavy reliance of participants on use assessment without investigating reuse. Participants felt that understanding how a digital object was reused played an important part in knowing the context and impact of that digital object. Relying on use statistics exclusively showed that an item could be discovered, but did not imply any additional meaning or significance. Participants felt that long-term use cases for collecting reuse data included better arguments for funding and justifying expense, more robust data to make digitization priorities, and increased ability to demonstrate outreach and community impact. Participants also noted concerns with privacy issues and cultural appropriation of digital objects.

\section{Technology and Standards Focus Groups}

The second round of focus groups, which took place in February and March of 2018, concentrated on the technology and standards needed to develop reuse assessment practices. Participants discussed the types of standards that should be implemented for measuring reuse, functional requirements of content management and repository systems to standardize or support reuse assessment, and new technologies needed to make cross-platform assessment feasible. They also discussed ethical and cultural implications of assessment and motivations for tracking reuse data.

One of the key outcomes of this discussion was the agreement that a single set of standards would not be effective. Participants recommended that a series of methodologies be created to meet specific key institutional goals. For instance, if there is a common desire within higher education for libraries to measure the impact of digital objects on learning outcomes, then a common methodology should be developed so that 
a library could (1) look to a recommended practice for learning outcomes assessment without having to reinvent the process and (2) be able to measure data in a standardized method across institutions. Likewise, if community archives were interested in citing how often their material was shared on disparate social media platforms in order to demonstrate community engagement, a potential reuse toolkit could include a common methodology for capturing and giving meaning to the impact of social media sharing. The key takeaway from this discussion was that assessment without purpose would simply add work but not value. Outcome-based assessment, driven by the goals of institutions, is the most effective use of time for CHKOs.

Additionally, these focus groups discussed the need to have data collection and analytics software that could work with content from multiple repositories and across multiple platforms (such as various social media venues), as well as integrate data from aggregators. They noted that the ability to build in reports and notification systems that acted in real time would allow them to engage with users. They also expressed concern at the use of third party analytics platforms that tracked user behavior and did not allow CHKOs more choice in protecting user privacy.

\section{Cultural and Privacy Implications Focus Groups}

The third set of in-person and virtual focus groups discussed questions about patron privacy and cultural issues from reuse of digital objects. Participants were asked what kinds of assessment data would not adversely impact privacy, local policies for protecting patron privacy, legal concerns, communities that would not benefit from assessment practices, how they approached culturally sensitive topics in their library, and the general ethical implications that are faced when doing assessment.

Participants reported a range of privacy policies in their institutions and the fact that these policies most often addressed in-person use of materials. They noted that reuse provides a different nuance than use statistics because the action is intentionally public and initiated by patrons, rather than by the institution. For instance, social media reuse of a digital object, by its nature, exposes personally identifiable information as it is a public-facing interaction, whereas initial use data is not intentionally public, and therefore comes with different expectations from the user. However, they felt that adhering to their institutions' current patron privacy policies, which prescribe destroying any personally identifiable information that is collected, would be advisable. They also noted that primarily utilizing aggregated reuse data could help alleviate some concern about the exposure of patron information. Participants felt that further examination and the development of conscientious standards would be a valuable tool. In addition, a toolkit with methods for negotiating privacy language in contracts with third party software vendors would be helpful.

When discussing ethical and cultural implications of reuse, participants were most concerned about reuse of collections by people, groups, or entities whose intentions were to malign the originating creators or subjects of collections. They felt that developing institutional policies around content reuse would be useful, and particularly that a potential toolkit could help provide examples that could be adopted or adapted as needed. With these kinds of policies in place, monitoring reuse would be less daunting when instances of sensitive cultural or ethical issues arose.

\section{Use Cases and Follow-up Survey}

Following the in-person and virtual focus groups, the results of the conversations were coded and analyzed for potential use cases. Three categories emerged. Data collection, analysis, and reporting use cases addressed how reuse data is being assembled, interpreted, and communicated to various audiences and stakeholders. Collection development use cases centered on how to assess aspects of a digital collection based on reuse data. Privacy, rights management, and ethics use cases focused on the assessment of reuse through legal, ethical, and culturally aware perspectives. Listed below are the use cases for each category. ${ }^{28}$

\section{Data Collection, Analysis, and Reporting:}

- Practitioner assesses quality and quantity of items reused from peer institutions for benchmarking

- Practitioner wants to tell stories of impact with the reuse data they have captured and tailor them to various audiences/stakeholders 
- Practitioner generates reports of collection item reuse for analysis

- Practitioner employs qualitative and quantitative methods to collect reuse data

- Practitioner understands how content is being reused in a variety of contexts by various audiences; contexts can include reuses on social media, in classrooms, in scholarly works, or through nonacademic avenues by audiences such as genealogists, digital humanists, teachers, and students

- Practitioner can find best practice documentation for collecting, analyzing, and reporting re-use data

- Practitioner is able to articulate the similarities and differences between use and reuse

\section{Collection Development:}

- Practitioner assesses quality and quantity of items reused to identify strengths and gaps in digital collections

- Practitioner assesses quality and quantity of items reused to inform digitization projects and priorities

- Practitioner updates existing access records with value-added content made by re-users, with attribution

- Practitioner develops new outreach services based on reuse data

- Practitioner implements DAMS/DLS with specific functionality for enabling reuse

Privacy, Rights Management, and Ethics:

- Practitioner wants to know who their users are without violating user privacy

- Practitioner can identify appropriative reuses of materials from marginalized communities

- Practitioner can identify platforms/venues where reuse content is occurring for commercial or marketing gain

- Practitioner wants to enable/encourage attribution of materials from their collection in various reuse contexts, including sharing and reposting on social media, integrating into classroom instruction, citing in scholarly works, or through non-academic avenues

- Practitioner provides ability for marginalized communities to report incidents of reuse that the community perceives as culturally exploitive

- Practitioner uses a code of ethics/conduct for assessing the reuse of content from diverse communities

The use cases outlined above were presented to the CHKO community via a follow-up survey ${ }^{29}$ conducted May 30 through June 15, 2018. Participants in the survey were asked to rank the use cases for each category in order to establish which use cases were the highest priority for practitioners. Along with these rankings, participants were also asked to prioritize 20 possible functional requirements for a toolkit, which would help determine the features a toolkit should include for greatest impact and utility. These functionalities were gathered from analyzing the focus group data as well as through a survey of existing toolkits. The options were: asynchronous training modules, best practices, blogs, examples of places or institutions implementing specific practices, an expertise or contact list, FAQs, forums, glossary of terminology, in-person trainings, links to outside resources, lists or links to tools and technologies, list or calendar of training opportunities, listserv, quick start guides and tutorials, resource library or list of readings, samples or templates (such as for reports or marketing), Slack channel integration, text-based guides or tutorials, video guides or tutorials, and webinars. The results from the 229 analyzed responses to the survey were finalized and reported in September 2018 and will provide the foundation for future development of a reuse assessment toolkit. ${ }^{30}$

\section{Practical Implications Prioritized Use Cases}

The follow-up survey results indicate that CHKO practitioners prefer resources, guidance, and tools that address increasing the awareness of assessment results and developing strategies for communicating these results to various stakeholders. The top five prioritized use cases were:

1. Understand how content is being reused in a variety of contexts by various audiences (social media, classrooms, scholarly works, genealogy, digital humanities, etc.) 
2. Tell stories of impact with the reuse data that has been captured and tailor it to specific audiences/stakeholders

3. Assess quality and quantity of items reused to inform digitization projects and priorities

4. Enable/encourage attribution of materials in various reuse contexts, including through sharing and reposting on social media, integration into classroom instruction, citing in scholarly works, or through non-academic avenues

5. Know and understand digital repository users without violating user privacy

Secondary use cases, while not as highly ranked by the community but still considered important, were:

1. Generate reports of collection item reuse for analysis

2. Employ qualitative and quantitative methods to collect reuse data

3. Assess quality and quantity of item reuse to identify strengths and gaps in digital collections

4. Provide ability for marginalized communities to report incidents of reuse that the community perceives as culturally exploitative

Collectively, these use cases suggest that any future toolkit should provide targeted content that emphasizes outcome-based assessment techniques, tools, and strategies. The prioritized use cases also indicate that the toolkit would not be complete without information that helps practitioners navigate privacy and cultural issues that are intertwined with better understanding and assessing content reuse.

\section{Prioritized Functional Requirements}

Overall, respondents to the survey indicated that they preferred best practices and quick start guides or tutorials above all other functional requirements. Following that, participants were also interested in samples or templates and examples of places or institutions implementing specific practices. Participants were least interested in features such as Slack channel integration, blogs, and email lists. This pointed to a noticeable professional interest in developing the theory and standards around reuse assessment first before engaging in practical discussions about how to employ those standards. These preferences might change in future years as reuse and assessment practices become more standardized. A future survey might be useful to pinpoint evolving preferences of the practitioner community.

When analyzing which functionalities best supported specific use cases, the high demand for the best practices, quick start guides, samples, templates, and example functionalities was consistently correlated across all use cases, with the exception of those participants who were highly interested in developing methods for encouraging attribution; they were more likely to request in-person trainings. Additionally, the responses from participants who reported being from institutions supporting underserved populations were examined independently from the general responses in order to determine what concrete steps could be taken to provide support for practitioners at these institutions. While they were also primarily interested in best practices, samples, and templates, they showed a significantly higher interest for in-person trainings and webinars. This was particularly true for tribal institutions (archives, libraries, museums, and research organizations) as well as institutions supporting Hispanic communities. In concert with the development of a reuse assessment toolkit, future efforts of the project team will also include an emphasis on in-person trainings and webinars to support these practitioners.

\section{Conclusion}

Existing literature and the authors' anecdotal experiences both indicate that practitioners are seeking better standards, methods, and tools to understand who uses digital repository content and for what reasons. 
CHKOs' participation in this project, through focus groups and surveys, suggest that a future toolkit, grounded in recommended assessment practices, should better prepare information professionals both for broad objectives (such as having access to strategies to tell impact stories about the reuse of collections) as well as for important cultural and privacy considerations.

Drawing upon the data presented in this paper, as well as content from "Barriers and solutions," the authors will continue the work of building a formalized content reuse assessment toolkit. The group has submitted a new grant application to IMLS, which, if awarded, would fund the development of the Digital Content Reuse Assessment Framework Toolkit (D-CRAFT). Should D-CRAFT not be funded, the authors will reevaluate the data and form a new plan for moving forward. As this paper indicates, there are numerous opportunities to improve the content reuse assessment landscape for practitioners and the authors are excited to be engaging CHKOs in this pursuit.

-Copyright 2019 Santi Thompson, Liz Woolcott, Caroline Muglia, Genya O'Gara, Ayla Stein Kenfield, and Elizabeth Joan Kelly

\section{Endnotes}

1. Kelly, et al.,"Setting a Foundation for Assessing Content Reuse.”

2. O'Gara et al., "Barriers and Solutions."

3. "Assessment:User/Reuse."

4. "Digital Library Assessment Interest Group."

5. Chapman et al., "Surveying the landscape."

6. Chapman et al., "Surveying the Landscape."

7. O'Gara et al., "Barriers and Solutions."

8. Chapman et al., "Surveying the Landscape," quoted in O'Gara et al., "Barriers and Solutions," 131.

9. Chapman et al., "Surveying the Landscape," quoted in O'Gara et al., "Barriers and Solutions," 131.

10. Chapman et al., "Surveying the Landscape," quoted in O'Gara et al., "Barriers and Solutions," 132.

11. Chapman et al., "Surveying the Landscape," quoted in O'Gara et al., "Barriers and Solutions," 131. Reverse image lookup articles include: Elizabeth Joan Kelly, "Reverse image lookup of a small academic library digital collection," Codex: the Journal of the Louisiana Chapter of the ACRL 3, no. 2 (2015): 80-92, quoted in O'Gara et al., "Barriers and Solutions," 132; Michele Reilly and Santi Thompson, "Reverse image lookup: assessing digital library users and reuses," Journal of Web Librarianship 11, no. 1 (2017): 56-68, quoted in O'Gara et al., "Barriers and Solutions," 132; Wikipedia citation tracking includes: Elizabeth Joan Kelly, "Use of Louisiana's Digital Cultural Heritage by Wikipedians," Journal of Web Librarianship 12, no. 2 (2018): 85-106, quoted in O'Gara et al., "Barriers and Solutions," 132; Google alert tracking includes: Elizabeth Joan Kelly, "Content Analysis of Google Alerts for Cultural Heritage Institutions," Journal of Web Librarianship 12, no. 1 (2018): 28-45, quoted in O'Gara et al., "Barriers and Solutions," 132;

Embedded metadata includes: Thompson and Reilly, "Embedded Metadata Patterns Across Web Sharing Environments, quoted in O'Gara, et al., "Barriers and Solutions," 132.

12. Punzalan, Marsh, and Cools, "Beyond Clicks," quoted in O'Gara et al., "Barriers and Solutions," 132.

13. Punzalan, Marsh, and Cools, "Beyond Clicks," quoted in O'Gara et al., "Barriers and Solutions," 132.

14. Xie, Joo, and Matusiak, "Multifaceted Evaluation."

15. "Xie, Joo, and Matusiak, "Multifaceted Evaluation," 8.

16. O'Gara et al., "Barriers and Solutions," 132.

17. O'Gara et al., "Barriers and Solutions," 138.

18. Thompson et al., "IMLS National Leadership Grant."

19. Thompson et al., "Developing a Framework."

20. For more information on the DLF Forum 2017, see: https://forum2017.diglib.org/.

21. For more information on Code4Lib 2018, see: https://2018.code4lib.org/. 
22. For more information on the Personal Digital Archiving Conference 2018, see: https://sites.lib.uh.edu/pda18/.

23. For more information on Dedoose, see: https://www.dedoose.com/.

24. O'Gara et al. "Barriers and Solutions."

25. Kelly et al., "Measuring Reuse of Digital Objects."

26. "Developing a Framework for the Reuse of Digital Objects: A Foundational Survey Analysis," found in Kelly et al., "Setting a Foundation."

27. "Developing a Framework for the Reuse of Digital Objects: A Foundational Survey Analysis," found in Kelly et al., "Setting a Foundation."

28. "Use Cases," found in Kelly et al., "Setting a Foundation."

29. "Developing a Framework for Measuring Reuse of Digital Objects: A Follow-up Survey," found in Kelly et al., "Setting a Foundation."

30. "Developing a Framework for the Reuse of Digital Objects: Follow-up Survey Analysis," found in Kelly et al., "Setting a Foundation."

\section{Bibliography}

Assessment Interest Group. “Assessment:User/Reuse.” Digital Library Federation. Last modified September 27, 2018. https://wiki.diglib.org/Assessment:User/Reuse.

Chapman, Joyce, Jody DeRidder, Megan Hurst, Elizabeth Joan Kelly, Martha Kyrillidou, Caroline Muglia, Genya O'Gara et al. Surveying the landscape: Use and usability assessment of digital libraries. OSF. April 19, 2016. https://osf.io/9nbqg. DOI 10.17605/OSF.IO/9NBQG.

"Digital Library Assessment Interest Group.” Digital Library Federation. Last modified September 27, 2018. https:/www.diglib.org/groups/assessment/.

Kelly, Elizabeth Joan. "Content Analysis of Google Alerts for Cultural Heritage Institutions." Journal of Web Librarianship 12, no. 1 (2018): 28-45.

Kelly, Elizabeth Joan, Caroline Muglia, Genya O'Gara, Ayla Stein, Santi Thompson, and Liz Woolcott. "Measuring Reuse of Digital Objects: Preliminary Findings from the IMLS-funded Project." In

Proceedings of the 18th ACM/IEEE on Joint Conference on Digital Libraries. ACM, 2018, 351-352.

Kelly, Elizabeth Joan. "Reverse image lookup of a small academic library digital collection." Codex: the Journal of the Louisiana Chapter of the ACRL 3, no. 2 (2015): 80-92.

Kelly, Elizabeth Joan, Ayla Stein Kenfield, Genya O’Gara, Caroline Muglia, Santi Thompson, and Liz Woolcott. "Setting a Foundation for Assessing Content Reuse: A White Paper From the Developing a Framework for Measuring Reuse of Digital Objects Project," September 24, 2018. https://doi.org/10.17605/OSF.IO/BQJVR.

Kelly, Elizabeth Joan. “Use of Louisiana's Digital Cultural Heritage by Wikipedians.” Journal of Web Librarianship 12, no. 2 (2018): 85-106.

O’Gara, Genya Morgan, Liz Woolcott, Elizabeth Joan Kelly, Caroline Muglia, Ayla Stein, and Santi Thompson. "Barriers and solutions to assessing digital library reuse: preliminary findings." Performance Measurement and Metrics 19, no. 3 (2018):130-141. https://doi.org/10.1108/PMM-03-2018-0012.

Punzalan, Ricardo L., Diana E. Marsh, and Kyla Cools. "Beyond Clicks, Likes, and Downloads: Identifying Meaningful Impacts for Digitized Ethnographic Archives." Archivaria 84, no. 1 (2017): 61-102. 
Reilly, Michele, and Santi Thompson. "Reverse image lookup: assessing digital library users and reuses." Journal of Web Librarianship 11, no. 1 (2017): 56-68.

Thompson, Santi, Elizabeth J Kelly, Caroline Muglia, Genya O'Gara, Ayla S. Kenfield, and Liz Woolcott. "Developing a Framework for Measuring Reuse of Digital Objects." OSF. July 2, 2018. DOI: osf.io/y5tjh.

Thompson, Santi, Elizabeth J Kelly, Caroline Muglia, Genya O'Gara, Ayla S Kenfield, and Liz Woolcott. "IMLS National Leadership Grant (LG-73-17-0002-17) 'Developing a framework for measuring reuse of digital objects.” IMLS. Last modified 2017. www.imls.gov/grants/awarded/lg-73-17-0002-17.

Thompson, Santi, and Michele Reilly. "Embedded Metadata Patterns across Web Sharing Environments." Presented at the $13^{\text {th }}$ International Digital Curation Conference (February 19-22, 2018). https://uhir.tdl.org/uh-ir/bitstream/handle/10657/3072/Embedded\%20Metadata\%20Patterns\%20Across \%20Web\%20Sharing\%20Environments.pdf?sequence=1.

Xie, Iris, Soohyung Joo, and Krystyna K. Matusiak. "Multifaceted Evaluation Criteria of Digital Libraries in Academic Settings: Similarities and Differences from Different Stakeholders." The Journal of Academic Librarianship 44, no. 6 (2018): 854-863. https://doi.org/10.1016/j.acalib.2018.09.002. 